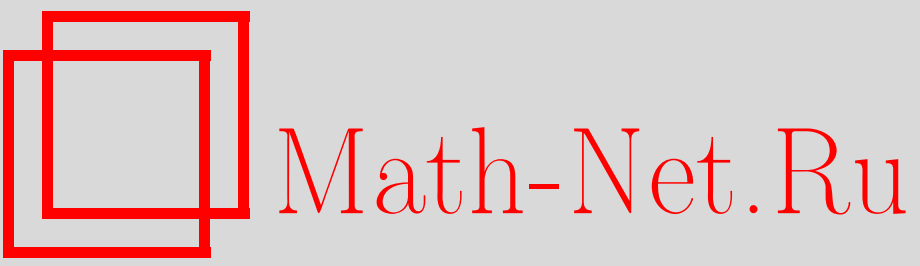

С. В. Людковский, Структура групп диффеоморфизмов неархимедовых многообразий, УМН, 2003, том 58, выпуск 6, 155-156

DOI: https://doi.org/10.4213/rm684

Использование Общероссийского математического портала Math-Net.Ru подразумевает, что вы прочитали и согласны с пользовательским соглашением

http://www.mathnet.ru/rus/agreement

Параметры загрузки:

IP: 34.227 .88 .159

26 апреля 2023 г., 10:40:52 


\title{
СТРУКТУРА ГРУПП ДИФФЕОМОРФИЗМОВ НЕАРХИМЕДОВЫХ МНОГООБРАЗИЙ
}

\author{
С.В. Людковский
}

Данная заметка посвящена структуре групп диффеоморфизмов (ГД) многообразий, как локально компактных, так и нелокально компактных, над неархимедовыми полями $\mathbf{K}$, где $\mathbf{C}_{\mathbf{p}} \supset$ $\mathbf{K} \supset \mathbf{Q}_{\mathbf{p}}$. Доказано, что ГД является простой и совершенной. Разделенные разности $\Phi^{n} f$ и их непрерывность определены для локально $\mathbf{K}$-вьпуклых пространств (ЛВП), где $0<n \in \mathbb{R}[1]$. С их помощью определяются различные классы гладкости групп $G(t, M):=C_{0}^{t, \mathrm{id}}(M) \cap \operatorname{Hom}(M)$ для многообразий $M$ на полных ЛВП, где $0 \leqslant t \leqslant \infty$. Они назьваются группами гомеоморфизмов при $0 \leqslant t<1$, а ГД при $1 \leqslant t \leqslant \infty[1]-[4]$. Для многообразия $M$, вложенного в шар радиуса $r$ нормированного пространства определена ГД $G\left(a n_{r}, M\right):=C_{0}^{a n_{r}, \mathrm{id}}(M) \cap \operatorname{Hom}(M)$ аналитических диффеоморфизмов. Класс $C_{0}^{t, \text { id }}$ отличается от $C^{t, \text { id }}$ условием, что он является пополнением семейства функций $f \mathrm{c} \operatorname{supp}\left(f_{i, j}-\mathrm{id}_{i, j}\right)$ и $\operatorname{range}\left(f_{i, j}-\mathrm{id}_{i, j}\right)$, конечномерными над $\mathbf{K}$ (сначала для ЛВП, а потом многообразий с помощью карт $\left(U_{j}, \phi_{j}\right)$ атласа $\left.\operatorname{At}(M)\right)$, где $f_{i, j}:=\phi_{i} \circ f \circ \phi_{j}^{-1}, U_{j}$ открыты в $M$.

Tеорема. Пусть группа $G:=G(t, M)$ задана для многообразия $M$, где $0 \leqslant t \leqslant \infty$ или $t=a n_{r}, r>0$. Тогда $G$ простая и совершенная группа.

Используя процедуру пополнения равномерного пространства, сведем доказательство к $G(t, M)$ с полными $M$ и $\mathbf{K}$.

(А) Рассмотрим сначала $G=G(t, M) \mathrm{c} t \geqslant 1$ или $t=a n_{r}$, а также открыто-замкнутую подгруппу $W$ из [1], [4], каждьй элемент которой принадлежит хотя бы одной однопараметрической локальной подгруппе. Если $f, g \in W$, то существуют векторные поля $A_{f}$ и $A_{g}$ класса $C_{0}^{t}$ на $M$ и однопараметрические подгруппы $f^{q}, g^{q} \subset W, q \in B(\mathbf{K}, 0,1)$, такие, что $\partial f^{q} / \partial q=A_{f} f^{g}$ и $\partial g^{q} / \partial q=A_{g} g^{q}$, где $A_{f}(x)=A_{f}^{i}(x) \partial_{i}$, суммирование ведется по $i \in I, I$ - направленное множество. Пусть $A^{i}$ класса $C_{0}^{\tau}$ с $\tau=\infty$ при $1 \leqslant t \leqslant \infty$ или $\tau=t$ при $t=a n_{r}$, тогда элементы вида $\exp (q A(x)) x$ плотны в $W$ при таких $t$, где $A=A^{i} \partial_{i}, q \in B(\mathbf{K}, 0,1)$. Для $B=\bar{A}^{i} \partial_{i}$ с $\bar{A}^{i}=\delta_{i, j}$, где $j \in I$ фиксировано, $\delta_{i, j}=1$ при $i=j$ и $\delta_{i, j}=0$ при $i \neq j \in I, W \ni \exp (q B) x \neq$ id. Если задано $C \in \operatorname{Vect}_{0}(\tau, M)$ c $\tau=\infty$ или $a n_{r},\|C\|_{\tau^{\prime}} \leqslant p^{-2}\left(\tau^{\prime} \in \mathbb{N}\right.$ или $\tau^{\prime}=a n_{r}$ соответственно), то существует $A \in \operatorname{Vect}_{0}(\tau, M)$ с $\|A\|_{\tau^{\prime}} \leqslant p^{-2}$ такое, что коммутатор векторных полей $[A, B]=C$. В самом деле, $[A, B]=A^{i}\left(\partial_{i} B^{k} \delta_{k, j}\right) \partial_{j}-B^{k} \delta_{k, j}\left(\partial_{j} A^{i}\right) \partial_{i}=-\left(\partial_{j} A^{i}\right) \partial_{i}=C^{i} \partial_{i}$. В силу $\S \S 79.1$ и 80.3 в [5] существует антидиффференцирование $P\left(x^{j}\right)$ по переменной $x^{j}$ (в локальных координатах атласа $\operatorname{At}(M))$ такое, что $A^{i}(x)=-\left(P\left(x^{j}\right) C^{i}\right)(x)$. Отсюда вытекает, что $[W, W]=W$, т.е. коммутант $[W, W]$ совпадает с $W$, где $[W, W]$ - минимальная подгруппа в $W$, порожденная подмножеством $\left\{[g, f]:=g \circ f \circ g^{-1} \circ f^{-1} \mid g, f \in W\right\}$. Таким образом, $W$ - совершенная группа.

Предположим, что имеется нормальная подгруппа $V$ в $W$, отличная от единичной $\{e\}$ и $W$, тогда $g V g^{-1}=V$ для любого $g \in W$. Пусть $v \subset w$ - соответствующие им подмножества в алгебре $\operatorname{Vect}_{0}(\tau, M)$, тогда $[v, w] \subset v$, где $[A, B]$ - коммутатор в алгебре $\operatorname{Vect}_{0}(\tau, M)$. Поэтому существуют $A \in w \backslash v$ и $0 \neq B \in v$. Поскольку $[v, w] \subset v$, то $\left[p^{2} \partial_{i}, B\right] \in v$ для любых $i$, т.е. можно полагать, что существуют $i \in I$ с $a\left(0, B^{i}\right) \neq 0$. Для $\operatorname{Vect}_{0}\left(a n_{r}, M\right)$ получим уравнения вида $\sum_{i, m+n=k+e_{i}}\left(b\left(n, C^{i}\right) b\left(m-e_{i}, B^{j}\right)-b\left(m, B^{i}\right) b\left(n-e_{i}, C^{j}\right)\right)=b\left(k, A^{j}\right)$, следователшно, решая их рекуррентно (в порядке возрастания $|m|, \operatorname{Ord}(m)$ и $j$ ), найдем $B \in v$ и $C \in w$, для которых $[C, B]=A$. Это возможно, так как для любого $c_{l}=p^{-l}, l \in \mathbb{N}$, семейство $\{(i,|n|<\operatorname{Ord}(n))$ : $\left.\left|b\left(n, A^{i}\right)\right| r^{|n|}>c_{l}\right\}$ конечно при $A \in \operatorname{Vect}_{0}\left(a n_{r}, M\right)$, где $m$-мулштииндекс, $b\left(k, A^{j}\right) \in \mathbf{K}$.

Локалшно аналитические функции [5] плотны в $C_{0}(t, M \rightarrow X)$ для остальных $1 \leqslant t \leqslant \infty$, следовательно, $[v, w]=w$ и получается противоречие. Таким образом, $W$ является простой группой, т.е. не содержит нормальных подгрупп $V$ с $V \neq\{e\}$ и $V \neq W$ одновременно. 
Группа $G$ представима в виде дизъюнктного объединения левых классов смежности $g_{i} W$, $G=\bigcup_{j \in \mathbb{N}} g_{j} W$, так что $\rho_{0}^{t}\left(g_{j}, g_{k}\right)>p^{-2}$ при $j \neq k$, следовательно, для выбранных $\left\{g_{j}: j \in \mathbb{N}\right\}$ с $g_{1}=e=\mathrm{id}$ и для любого $f \in G \backslash W$ существуют единственные $j$ и $f_{2} \in W$ в $f=g_{j} f_{2}$. Из $\bar{Q}_{m}(x+c)=\bar{Q}_{m}(x)$ при $|m|>0$ и всех $x \in B(\mathbf{K}, 0,1)$ следует, что $c=0$, где $n=\operatorname{Ord}(m), \bar{Q}_{m}$ - базисные полиномы Амис. Тогда, перебирая $g \in W$ вида (id $\left.+\xi \bar{Q}_{m}(x) e_{i}\right), \xi \in B\left(\mathbf{K}, 0, p^{-2}\right)$, получим, что $[G \backslash W, W] \supset G \backslash W$ и $\left\{g f g^{-1}: g \in W\right\} \neq\{f\}$ для любых $f \in G \backslash W$, следовательно, $[G, G]=G$.

Пусть имеется нормальная подгруппа $V \subset G, V \neq e, V \neq G$. Тогда для любых $f$ и $g \in V$ с $f g^{-1} \in W$ получим, что $f g^{-1}=e$, так как $V \cap W$ является нормальной подгруппой в $W$, следовательно, $V$ дискретна и $\rho_{0}^{t}(f, g)>p^{-2}$ для любых $f \neq g \in V$. Тогда $h f h^{-1}=f$ для любых $h \in W$, но это противоречит приведенньм вьше рассуждениям. Таким образом, $G$-простая совершенная группа.

(В) При $0 \leqslant t<1$ группа $G(1, M)$ плотна в топологической группе $G(t, M)$, следовательно, $G(t, M)$ также проста и совершенна.

Общий случай для многообразий на ЛВП получается с помощью того, что ЛВП является проективньм переделом нормированных пространств, что дает также разложение функций в пределы обратных спектров функций на нормированных пространствах, а потом, используя карты, получаем $W$. При этом $W$ замкнуто в тихоновской и открыто-замкнуто в ящичной топологиях соответственно, наследуемых проективным пределом как подмножеством произведения и т. д. в каждой из этих топологий (см. подробнее [4]).

\section{СПИСОК ЛИТЕРАТУРЫ}

[1] S. V. Ludkovsky // Ann. Math. Blaise Pascal. 2000. V. 7. № 2. P. 19-80. [2] C. В. Людковский // Теорет. и матем. физика. 1999. Т. 119. № 3. С. 698-711. [3] С. В. Л юдковский // УМH. 1996. T. 51. № 2. C. 169-170. [4] S. V. Ludkovsky. A structure and representations of diffeomorphism groups of non-Archimedean manifolds. Preprint. Los Alamos: Los Alamos Nat. Lab. math.GR/0004126, 2000. [5] W. H. Schikhof. Ultrametric Calculus. Cambridge: Cambridge Univ. Press, 1984. 